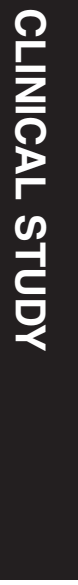

${ }^{1}$ Department of Ophthalmology, Eye and ENT Hospital, Shanghai Medical College, Fudan University, Shanghai,

China

${ }^{2}$ Department of Ophthalmology, No. 5 People's Hospital of Shanghai, Shanghai, China

${ }^{3}$ Shanghai Key Laboratory of Visual Impairment and Restoration; Key Laboratory of Myopia of State Health Ministry,

Fudan University,

Shanghai, China

Correspondence:

$\mathrm{CH}$ Jiang, Department of Ophthalmology, Eye and ENT Hospital, Shanghai Medical College, Fudan University, Shanghai 200031, China or Department of Ophthalmology, No. 5 People's Hospital of Shanghai, Shanghai 200240, China Tel: +86 13801843 682; Fax: +862164370134. E-mail: chhjiang70@163. com

${ }^{4}$ These authors contributed equally to this study.

Received: 14 November 2016

Accepted in revised form: 16 October 2017

Published online:

17 November 2017

\section{Differences in photoreceptor recovery among patients and between different parts of the posterior pole in Vogt- Koyanagi-Harada disease}

\begin{abstract}
Purpose To investigate the recovery of photoreceptors following the treatment in Vogt-Koyanagi-Harada (VKH) disease. Patients and methods This was a retrospective study. We enrolled 28 patients with VKH (56 eyes). The clinical and optical coherence tomography (OCT) findings were recorded for 12 months after treatment. The patterns of photoreceptor recovery on OCT were defined: pattern $\mathrm{F}$ group $=$ Foveal photoreceptor recovery visible first; pattern E group $=$ Extrafoveal photoreceptor recovery visible first; and pattern $S$ group = Simultaneous foveal and extrafoveal photoreceptor recovery.

Results Photoreceptor recovery varied in different parts of the fundus among patients. Among the 56 eyes, the ellipsoid zone (EZ) recovery of $\mathbf{1 0}$ eyes and the interdigitation zone (IZ) recovery of 17 eyes belonged to pattern $\mathrm{F}$ group. In most eyes (46 eyes for EZ and 26 eyes for IZ), the recovery of these structures were pattern S. Only in 10 eyes, the recovery of IZ was pattern $E$. The different patterns of recovery correlated with how promptly the patients had been treated and with the anatomical and visual outcomes at 12 months. Patients in pattern $\mathrm{F}$ group were characterized by delayed treatment, delayed recovery of $\mathrm{EZ}$ or $\mathrm{IZ}$, and a less favourable prognosis at $\mathbf{1 2}$ months relative to other

Eye (2018) 32, 572-578; doi:10.1038/eye.2017.250; published online 17 November 2017

Introduction

Vogt-Koyanagi-Harada (VKH) disease is a granulomatous inflammatory disorder that affects the eyes, auditory system, meninges, and skin. It accounts for about $6.7-16 \%$ of all cases of uveitis in East Asia. ${ }^{1,2}$ Spectral-domain optical coherence tomography (SD-OCT) studies have revealed morphological changes, including choroidal thickening, undulation of the retinal pigment epithelium (RPE), subretinal fluid accumulation, and blurring of the photoreceptor ellipsoid zone (EZ), in the eyes of patients with acute VKH disease. ${ }^{3,4}$ The manifestations of latestage VKH disease that are detected with OCT include RPE atrophy, loss of EZ, loss of the external limiting membrane, loss of the outer nuclear layer, and choroidal thinning. 5,6

However, little is known about the changes that occur between the acute and late stage. We previously reported that some late-stage VKH eyes had a fragmented EZ or interdigitation zone (IZ), but these structures were intact in the central fovea. ${ }^{7}$ Therefore, we performed this study to evaluate the recovery of photoreceptors in patients with acute VKH disease, and to explore whether the photoreceptors in different parts of the fundus recovered differently.
\end{abstract} patients, while those in pattern E group had the most prompt treatment and recovery as well as a more favourable outcome at $\mathbf{1 2}$ months. Conclusions In VKH patients with delayed treatment, foveal photoreceptors tended to recover more rapidly than photoreceptors in other regions.

\author{
M Zhou ${ }^{1,4}, \mathrm{RP} \mathrm{Gu}^{1,4}, \mathrm{Z}$ Sun ${ }^{1}, \mathrm{CH}$ Jiang ${ }^{1,2}$, \\ Q Chang ${ }^{1}$ and $\mathrm{GZ} \mathrm{Xu}^{1,3}$
}

Methods

Study participants

We reviewed the medical records of patients diagnosed with VKH disease and who received 
standard corticosteroid treatment at the Department of Ophthalmology, Eye and ENT Hospital, Fudan University, between January 2013 and April 2015. VKH disease was diagnosed according to the revised diagnostic criteria proposed by the International Nomenclature Committee. ${ }^{3}$ The standard corticosteroid therapy defined as starting with steroid pulse (intravenous drip infusion of $1 \mathrm{~g}$ methylprednisolone/day for three consecutive days) followed by a $1 \mathrm{mg} / \mathrm{kg}$ daily dose of oral prednisolone. The dose of oral prednisolone was gradually tapered over more than 6 months. Beginning with low-dose of oral prednisolone is defined as nonstandard treatment. If there was a relapse during the tapering of oral prednisolone, the dosage was increased or adjuvant cyclosporine was administered as needed at a dose of $3 \mathrm{mg} / \mathrm{kg} /$ day; and then the dose was tapered according to the severity of the ocular inflammation. The study conformed to the tenets of the Declaration of Helsinki, and was approved by the Institutional Review Board of the Eye and ENT Hospital of Fudan University. All of the subjects signed an informed consent form.

\section{Data collection}

Data were recorded at visits before treatment and at 1, 3, 6, and 12 months after treatment. Each visit involved a comprehensive ophthalmological examination, including the assessment of the best corrected visual acuity (BCVA), assessment of retinal sensitivity with a microperimeter (except at the first visit), measurement of intraocular pressure, slit-lamp biomicroscopy, fundoscopic examination with a noncontact lens, and SD-OCT.

\section{Microperimetry}

After pupil dilation, all patients were tested with microperimetry using an MP-1 microperimeter (software version SW1.4.1 SP1; Nidek Technologies, Padua, Italy). The settings included a Goldmann III-sized target and the 4-2 staircase strategy, with 45 stimulated locations within a diameter of $12^{\circ} .7$ Microperimetry was performed by an experienced ophthalmologist (ZS) who was masked to the patient's medical history. The software automatically calculated the retinal sensitivity, which was defined as the mean of all 45 scores. The retina was then divided into two regions: the central foveal area (13 central spots covering $0-4^{\circ}$ ) and the peripheral area (the remaining 32 spots in an annulus of $5-12^{\circ}$ ) (Supplementary Figure S1).

\section{Spectral-domain optical coherence tomography}

After the pupil was fully dilated, EDI-OCT images were captured with a Spectralis HRA+OCT version 1.5.12.0
(Heidelberg Engineering, Heidelberg, Germany) in the volume scan mode, which covered a $30^{\circ} \times 30^{\circ}$ region centred on the fovea $(1024 \times 25 ; 25$ lines). Only scans with good signal strength (signal strength $\geq 20$ ) were used for the analysis. The OCT images were taken before treatment and at 1, 3, 6 and 12 months after treatment. The follow-up function on OCT was used to evaluate the same points. A horizontal scan line crossing the centre of the fovea was selected for further analysis. The OCT images were graded using a standardized protocol by an experienced ophthalmologist who was masked to the patient's characteristics and the date of follow-up. To determine whether the photoreceptors within and outside the fovea recovered differently, EZ and IZ within and outside the fovea were graded separately. The patterns of photoreceptor recovery on OCT were defined like this: pattern $\mathrm{F}$ group = Foveal photoreceptor recovery visible first; pattern E group = Extrafoveal photoreceptor recovery visible first; and pattern $S$ group $=$ Simultaneous foveal and extrafoveal photoreceptor recovery. The fovea was defined as the central $1.5 \mathrm{~mm}$ region of the macular on each cross-section scan. OCT grading at the 1-, 3-, and 6-month visits recorded whether the photoreceptors were visible. Using images obtained at 12 months, the photoreceptors were graded as intact or not (Supplementary Figure S2).

\section{Statistical analysis}

The BCVA was converted to logarithms of the minimum angle of resolution (logMAR) for statistical analysis. The linear mixed model was performed to compare the differences in retinal sensitivity, BCVA and the recovery of out retinal structures. In all analyses, values of $P<0.05$ were considered statistically significant. All analyses were performed with SPSS for Windows version 17.0 (SPSS, Inc., Chicago, IL, USA).

\section{Results}

A total of 28 patients with VKH disease (11 males, 17 females; 56 eyes) attended all follow-up visits at 1, 3, 6, and 12 months, and were included in the study. At the first visit, the mean age of the patients was $34.14 \pm 12.49$ years, the mean BCVA (logMAR) was $0.84 \pm 0.52$ (Supplementary Table S1). Ten patients (20 eyes) had previously received non-standard corticosteroid therapy, and the mean time from the onset of symptoms to treatment (the standard treatment starting at our hospital) was $18.96 \pm 16.39$ days.

After treatment, the BCVA and mean retinal sensitivity improved markedly (Supplementary Figure S3). At 12 months, the mean BCVA $(\log M A R)$ was $0.07 \pm 0.09$ and the microperimetric sensitivity was $18.46 \pm 1.79 \mathrm{~dB}$. 
Retinal detachment resolved quickly. At 1 month after treatment, 11 (19.64\%) eyes had evidence of retinal detachment on OCT image, and in nine of them, the retinal detachment was localized to the central macular region (Supplementary Figure S4a). All the retinal detachments had resolved by 3 months. The EZ and IZ were gradually reappeared after treatment. At 1 month, 76.78 of EZ and $48.21 \%$ of IZ had reappeared on OCT images. The ratio increased to 94.64 for $\mathrm{EZ}$ and $71.42 \%$ for IZ by 3 months. At 12 months, all eyes (100\%) showed visible EZ and $47(83.93 \%)$ eyes showed visible IZ. The IZ of nine eyes $(16.07 \%)$ had not recovered at the end of 12 months.

Although EZ and IZ became visible after treatment, there were different patterns in their recovery. Among the 56 eyes, the EZ recovery of 10 eyes (Figure 1a) and the IZ recovery of 17 eyes (Figure $2 \mathrm{a}$ ) belonged to pattern $\mathrm{F}$ group. In most eyes (46 eyes for EZ and 26 eyes for IZ), the recovery of these structures were pattern $S$ (Figures $1 b$ and $2 b)$. Only in 10 eyes, the recovery of IZ was pattern E (Figure 2c). And no case of EZ recovery was pattern E.

The age and sex of patients in pattern $\mathrm{F}$ group were similar to those in pattern $S$ or pattern E group. However, the eyes in pattern $\mathrm{F}$ group were associated with worse initial vision, delayed treatment, delayed recovery of EZ or IZ, and worse anatomical and visual outcomes at 12 months (Tables 1 and 2). Eyes in pattern $S$ group had better initial vision, received prompt treatment, showed faster recovery of EZ or IZ, and had better anatomical and visual outcomes at 12 months (Tables 1 and 2). The 10 eyes in pattern $\mathrm{E}$ group were treated most rapidly, with a mean of 9.8 days after symptom onset (Table 2 ). The differences in clinical variables among the groups of eyes were still significant when patients who had previously received non-standard treatment were excluded from the analysis (Table 3). Compared with patients in pattern $S$ group, patients in pattern $\mathrm{F}$ group were characterized by delayed treatment, delayed recovery of IZ, and worse retinal sensitivity at 12 months after treatment (Table 3).

\section{Discussion}

Many studies had proved that the reappearance of EZ or IZ could be considered as the site of photoreceptor recovery in many retinal diseases. ${ }^{8-10}$ In this study, we describe the recovery of photoreceptors, determined by the reappearance of EZ or IZ on SD-OCT, after standard
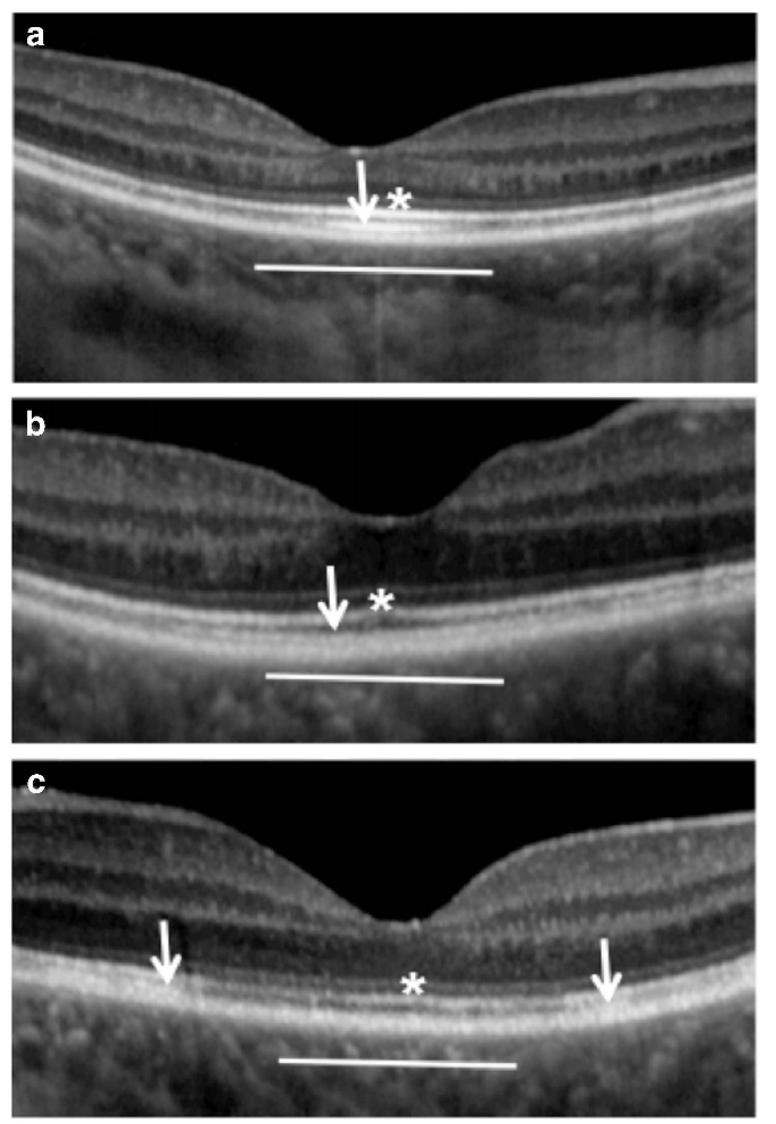

Figure 2 Different patterns of recovery in the interdigitation zone (IZ). Representative images showing (a) pattern F for IZ: Foveal IZ recovery visible first, (b) pattern S for IZ: Simultaneous foveal and extrafoveal IZ recovery, and (c) pattern E for IZ: Extrafoveal IZ recovery visible first. Lines indicate the macular region $(1.5 \mathrm{~mm})$. The arrow as IZ and asterisk as EZ.
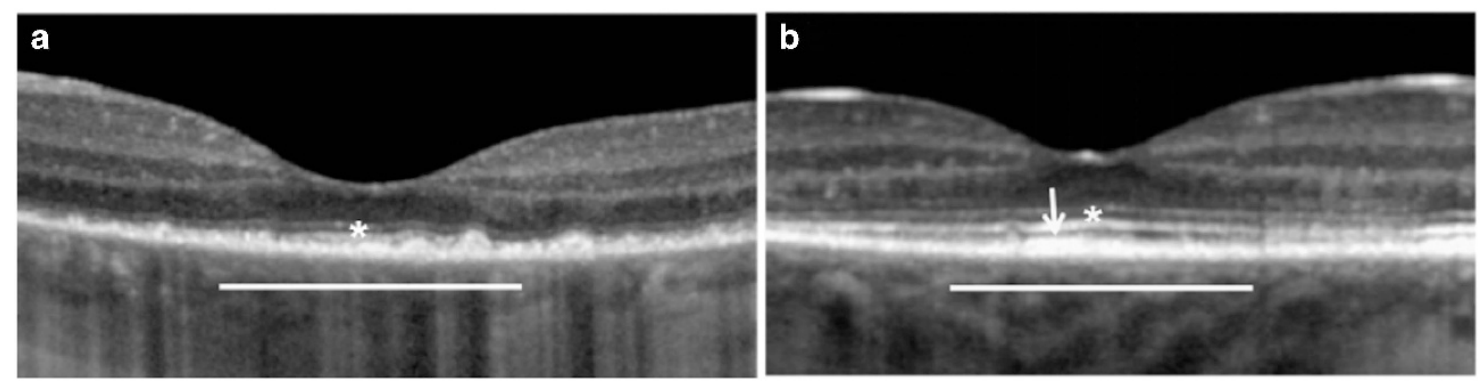

Figure 1 Different patterns of recovery in the ellipsoid zone (EZ). Representative images showing (a) pattern F for EZ: Foveal EZ recovery visible first, and (b) pattern S for EZ: Simultaneous foveal and extrafoveal EZ recovery. Lines indicate the macular region $(1.5 \mathrm{~mm})$. The arrow as IZ and asterisk as EZ. 
Table 1 Characteristics of patients according to the pattern of ellipsoid zone (EZ) recovery

\begin{tabular}{|c|c|c|c|}
\hline & \multicolumn{2}{|c|}{ EZ recovery pattern } & \multirow[t]{2}{*}{ P-value } \\
\hline & Pattern F group & Pattern S group & \\
\hline Number of eyes & $10(17.9 \%)$ & $46(82.2 \%)$ & \\
\hline Sex (male) & $2(20.0 \%)$ & $20(43.4 \%)$ & 0.178 \\
\hline Age (years) & $35.0 \pm 13.7$ & $33.9 \pm 12.4$ & 0.815 \\
\hline BCVA (logMAR) before treatment & $1.07 \pm 0.45$ & $0.79 \pm 0.53$ & 0.132 \\
\hline Time from symptom onset to treatment (days) & $34.9 \pm 16.7$ & $15.5 \pm 14.3$ & $<0.0001$ \\
\hline Time from treatment to recovery of EZ (months) & $4.5 \pm 4.2$ & $1.1 \pm 0.5$ & $<0.0001$ \\
\hline Time from treatment to recovery of IZ (months) & $6.0 \pm 3.0$ & $2.3 \pm 1.7$ & $<0.0001$ \\
\hline \multicolumn{4}{|l|}{ Findings at 12 months } \\
\hline BCVA (logMAR) & $0.12 \pm 0.13$ & $0.05 \pm 0.07$ & 0.036 \\
\hline Retinal sensitivity $(\mathrm{dB})$ & $16.1 \pm 2.2$ & $18.9 \pm 1.2$ & $<0.0001$ \\
\hline Eyes with intact EZ & $2(20.0 \%)$ & $41(89.1 \%)$ & $<0.0001$ \\
\hline Eyes with intact foveal EZ & $8(80.0 \%)$ & $46(100.0 \%)$ & 0.001 \\
\hline Eyes with intact IZ & $0(0 \%)$ & $19(41.3 \%)$ & 0.012 \\
\hline Eyes with intact foveal IZ & $2(20.0 \%)$ & $33(71.7 \%)$ & 0.002 \\
\hline
\end{tabular}

Abbreviations: EZ, ellipsoid zone; IZ, interdigitation zone; BCVA, best corrected visual acuity; logMAR, logarithm of the minimum angle of resolution. The linear mixed model was performed to compare the differences between groups. $P<0.05$ was considered statistically significant. Values are presented as $n(\%)$ or means \pm standard deviations. Pattern F group =Foveal EZ recovery visible first; pattern E group = Extrafoveal EZ recovery visible first; and pattern $\mathrm{S}$ group $=$ Simultaneous foveal and extrafoveal EZ recovery.

Table 2 Characteristics of patients according to the pattern of interdigitation zone (IZ) recovery

\begin{tabular}{|c|c|c|c|c|}
\hline & \multicolumn{3}{|c|}{ IZ recovery pattern } & \multirow{2}{*}{ P-value } \\
\hline & Pattern F group & Pattern S group & Pattern E group & \\
\hline Number of eyes & $17(32.1 \%)$ & $26(49.1 \%)$ & $10(18.9 \%)$ & \\
\hline Sex (male) & $5(29.41 \%)$ & $13(50 \%)$ & $4(40 \%)$ & 0.284 \\
\hline Age (years) & $29.4 \pm 13.7$ & $35.4 \pm 11.4$ & $35.9 \pm 10.5$ & 0.172 \\
\hline BCVA (logMAR) before treatment & $0.91 \pm 0.55$ & $0.76 \pm 0.50$ & $0.50 \pm 0.50$ & 0.64 \\
\hline Time from symptom onset to treatment (days) & $26.9 \pm 16.0$ & $13.2 \pm 10.3$ & $9.8 \pm 11.4$ & $<0.0001$ \\
\hline Time from treatment to recovery of EZ (months) & $1.9 \pm 1.4$ & $1.1 \pm 0.4$ & $1.0 \pm 0.0$ & $<0.0001$ \\
\hline Time from treatment to recovery of IZ (months) & $4.9 \pm 2.4$ & $2.0 \pm 1.5$ & $1.0 \pm 1.0$ & $<0.0001$ \\
\hline \multicolumn{5}{|l|}{ Findings at 12 months } \\
\hline BCVA (log MAR) & $0.07 \pm 0.08$ & $0.03 \pm 0.06$ & $0.06 \pm 0.07$ & 0.21 \\
\hline Retinal sensitivity $(\mathrm{dB})$ & $17.6 \pm 1.9$ & $19.1 \pm 0.9$ & $19.5 \pm 0.7$ & 0.001 \\
\hline Eyes with intact EZ & $9(52.94 \%$ & $24(92.31 \%)$ & $10(100 \%)$ & 0.001 \\
\hline Eyes with intact foveal EZ & $17(100 \%)$ & $26(100 \%)$ & $10(100 \%)$ & 1.0 \\
\hline Eyes with intact IZ & $1(5.9 \%)$ & $14(53.9 \%)$ & $4(40 \%)$ & 0.006 \\
\hline Eyes with intact foveal IZ & $5(29.4 \%)$ & $22(84.6 \%)$ & $8(80 \%)$ & 0.001 \\
\hline
\end{tabular}

Abbreviations: EZ, ellipsoid zone; IZ, interdigitation zone; BCVA, best corrected visual acuity; logMAR, logarithm of the minimum angle of resolution. The linear mixed model was performed to compare the differences between groups. $P<0.05$ was considered statistically significant. Values are presented as $n(\%)$ or means \pm standard deviations. Pattern F group = Foveal IZ recovery visible first; pattern E group = Extrafoveal IZ recovery visible first; and pattern $S$ group $=$ Simultaneous foveal and extrafoveal IZ recovery.

corticosteroid treatment in patients with acute VKH disease. The patterns of photoreceptor recovery varied among patients and in different parts of the posterior pole. In most eyes, these structures reappeared simultaneously within and outside the fovea, while in some eyes, the EZ and IZ reappeared firstly within the fovea and in some firstly outside the fovea. These different patterns of recovery were associated with how promptly the patients had been treated and with the visual or anatomical prognosis at 12 months. As well as delayed treatment, preoperative pathology such as the presence of choroidal undulation and subretinal fluid were linked to a delay in recovery of the outer retina. Compared with the eyes with choroidal undulations before treatment, the ones without choroidal undulations showed faster recovery of EZ/IZ, and had better retinal function at 12 months (Supplementary Table S2). And also the ones without subretinal fluid before treatment 


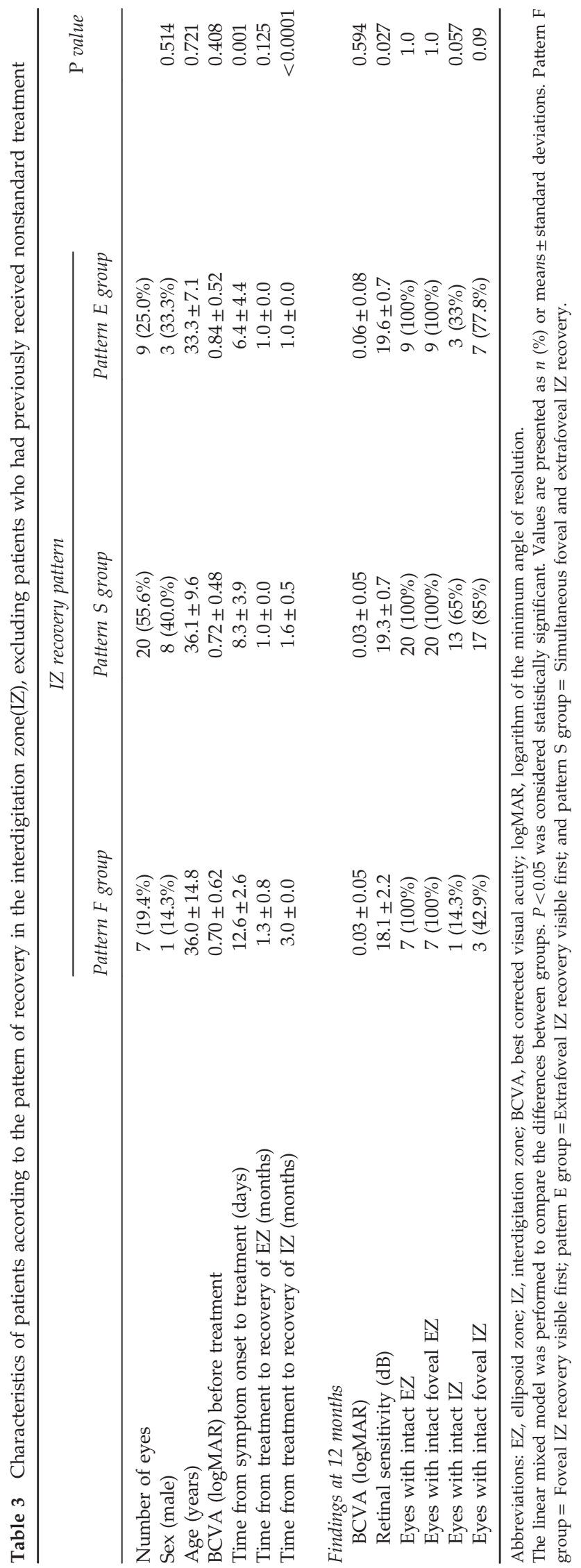


had better initial vision, showed faster recovery of IZ, and had better anatomical at 12 months than eyes with subretinal fluid (Supplementary Table S3).

In our result, in 9 of the 11 eyes with retinal detachment at 1 month, the detachment was localized only at the central macular region, and in the other two beside the localized retinal detachment at the central macula, there was only slight subretinal fluid around the disc (Supplementary Figure S4). Patients with acute VKH disease commonly display macular retinal detachment. However, it is unclear whether this retinal detachment originates from the macular region. Our data at least indicated that retinal detachment in the central macular region was the last to resolve. The 10 eyes in which the photoreceptors in IZ recovered first outside the fovea had the shortest time from the onset of symptoms to treatment (mean 9.8 days), and a quick recovery (the mean time from treatment to the finding of IZ $1 \pm 1$ month) (Supplementary Figure S5). The reason might be as follows. In these patients, because of prompt treatment, the retinal detachment might exist for a shorter period of time, and as the retinal detachment outside the fovea resolved first, the detachment outside the fovea lasted for even shorter period of time. So in these eyes the photoreceptors outside the fovea might have been mildly impaired, thus allowing fast recovery.

In most eyes, EZ or IZ (46/56 for EZ and 26/56 for IZ) were found simultaneously at and outside the fovea, and in some eyes they (10 for EZ and 17 for IZ) were found firstly at the fovea, despite the fact that the retinal detachment at the fovea resolved last. This might result from the high density of cones in the fovea, ${ }^{11}$ which made it easier to detect their reappearance or recovery. Also the foveal RPE is taller and contains more melanosomes than the RPE in other regions. ${ }^{11}$

What is more interesting, analysis found that eyes with photoreceptors found first at the fovea seemed to have a significantly delayed treatment, and this difference was still significant when we excluded patients who had previously received non-standard treatment (Table 3). And in nine eyes with localized foveal detachment at 1 month, the EZ in four eyes and IZ in five eyes recovered first within the fovea (Supplementary Figure S4b,c). Also in our former study of late stage VKH eyes, we found that in eyes with EZ or IZ defects outside the fovea, these structures could remain intact at the fovea, but in eyes with EZ or IZ defects at the fovea there were always defects in these structures outside the fovea. ${ }^{7}$ These might suggest that at the fovea, beside the high density of the photoreceptor and the taller RPE, the photoreceptors there might be either resistant to VKH-related inflammation or might recover much better than photoreceptors outside the fovea. One more thing, formerly Curcio reported that the photoreceptor density of human was highly variable between individuals, ${ }^{12}$ such variation might also exist in the structure or density of RPE as well. These foveal structures might also be relevant to the pattern of recovery of outer retinal structure.

Patients whose EZ or IZ outside the fovea recovered last were characterized by delayed treatment and worse anatomic and visual outcomes at 12 months. This association between delayed treatment and worse outcomes strengthens the importance of prompt standardized treatment for VKH. And at the end of 12month follow-up, the BCVA and retinal sensitivity were comparable to healthy subjects (BCVA logMAR $0.07 \pm 0.09$ and retinal sensitivity $18.46 \pm 1.79 \mathrm{~dB}) .{ }^{13}$ So with standard treatment, VKH patients could achieve a rather good visual and structural outcome.

The exact reason for the comparatively rapid and good recovery of photoreceptors in the fovea in patients with a delayed treatment is unclear. Therefore, further studies are required to understand the reason for this. Such studies might reveal new ways to improve the prognosis of $\mathrm{VKH}$ and other retinal diseases, especially macular diseases.

In conclusion, OCT findings over 12 months after treatment revealed that the recovery of photoreceptor differed among the patients, and different patterns were associated with how prompt the treatment was. And in patients for whom treatment was delayed, the photoreceptors at the fovea seemed to recover more rapidly and better than those in the peripheral regions of eyes.

Summary

What was known before

- The recovery of photoreceptors in patients with VKH disease was unclear.

What this study adds

- In VKH patients with delayed treatment, foveal photoreceptors tended to recover more rapidly than photoreceptors in other regions.

\section{Conflict of interest}

The authors declare no conflict of interest.

\section{Acknowledgements}

This study was supported in part by research grants from the National Natural Science Foundation of China (81570854), the National Key Basic Research Program of China (2013CB967503), the National Major Scientific Equipment Program (grant no. 2012YQ12008003), and the 
National Key Basic Research Program of China (grant no. 2013CB967503).

\section{References}

1 Yang P, Ren Y, Li B, Fang W, Meng Q, Kijlstra A. Clinical characteristics of Vogt-Koyanagi-Harada syndrome in Chinese patients. Ophthalmology 2007; 114(3): 606-614.

2 Goto H, Mochizuki M, Yamaki K, Kotake S, Usui M, Ohno S. Epidemiological survey of intraocular inflammation in Japan. Jpn J Ophthalmol 2007; 51(1): 41-44.

3 Read RW, Holland GN, Rao NA, Tabbara KF, Ohno S, Arellanes-Garcia L et al. Revised diagnostic criteria for VogtKoyanagi-Harada disease: report of an international committee on nomenclature. Am J Ophthalmol 2001; 131(5): 647-652.

4 Tsuboi K, Nakai K, Iwahashi C, Gomi F, Ikuno Y, Nishida K. Analysis of choroidal folds in acute Vogt-Koyanagi-Harada disease using high-penetration optical coherence tomography. Graefes Arch Clin Exp Ophthalmol 2015; 253(6): 959-964.

5 Lee EK, Lee SY, Yu HG. A clinical grading system based on ultra-wide field retinal imaging for sunset glow fundus in Vogt-Koyanagi-Harada disease. Graefes Arch Clin Exp Ophthalmol 2015; 253: 359-368.

6 Takahashi $\mathrm{H}$, Takase $\mathrm{H}$, Ishizuka A, Miyanaga M, Kawaguchi T, Ohno-Matsui $\mathrm{K}$ et al. Choroidal thickness in convalescent vogt-koyanagi-harada disease. Retina 2014; 34 (4): 775-780.

7 Zhou M, Jiang C, Gu R, Sun Z, Huynh N, Chang Q. Correlation between retinal changes and visual function in late-stage Vogt-Koyanagi-Harada disease: an optical coherence tomography study. J Ophthalmol 2015; 2015: 916485.

8 Itoh Y, Inoue M, Rii T, Hiraoka T, Hirakata A. Significant correlation between visual acuity and recovery of foveal cone microstructures after macular hole surgery. Am J Ophthalmol 2012; 153(1): 111-119.

9 Hirota K, Itoh Y, Rii T, Inoue M, Hirakata A. Correlation between foveal interdigitation zone band defect and visual acuity after surgery for macular pseudohole. Retina 2015; 35(5): 908-914.

10 Shimozono M, Oishi A, Hata M, Matsuki T, Ito S, Ishida K et al. The significance of cone outer segment tips as a prognostic factor in epiretinal membrane surgery. Am J Ophthalmol 2012; 153(4): 698-704, 701-704.

11 Spaide RF, Curcio CA. Anatomical correlates to the bands seen in the outer retina by optical coherence tomography: literature review and model. Retina 2011; 31(8): 1609-1619.

12 Curcio CA, Sloan KR, Kalina RE, Hendrickson AE. Human photoreceptor topography. J Comp Neurol 1990; 22(4): 497-523.

13 Springer C, Volcker H E, Rohrschneider K. Static fundus perimetry in normals. Microperimeter 1 versus SLO. Ophthalmologe 2006; 3(3): 214-220.

(c) (1) (2) $\odot$ This work is licensed under a Creative Commons Attribution-NonCommercialNoDerivs 4.0 International License. The images or other third party material in this article are included in the article's Creative Commons license, unless indicated otherwise in the credit line; if the material is not included under the Creative Commons license, users will need to obtain permission from the license holder to reproduce the material. To view a copy of this license, visit http://creativecommons.org/licenses/by-nc-nd/4.0/

(C) The Author(s) 2018

Supplementary Information accompanies this paper on Eye website (http:/ /www.nature.com/eye) 